\title{
Atendimentos prestados pelo serviço móvel de urgência em Cuiabá, MT, Brasil ${ }^{1}$
}

\author{
Services rendered by the mobile emergency service of Cuiabá, MT, Brazil
}

Servicios prestados por la emergencia móvil en Cuiabá, MT, Brasil

Sebastião Junior Henrique Duarte ${ }^{\mathrm{I}}$, Bruno Brandão de Lucena ${ }^{\mathrm{II}}$, Lia Hanna Martins Morita ${ }^{\mathrm{II}}$

\section{RESUMO}

Este estudo teve como objetivo caracterizar os atendimentos prestados pelo Serviço de Atendimento Móvel de Urgência do município de Cuiabá - MT, nos seis primeiros meses de funcionamento. Estudo descritivo, baseado em análise documental do sistema de informação do SAMU de Cuiabá, realizado de fevereiro a junho de 2009 . Foram descritos 1893 atendimentos, sendo 1293 devidos a causas traumáticas e 578 a causas clínicas. Dos atendimentos devidos a causas clínicas, $51,2 \%$ foram prestados a mulheres; dentre aqueles com causas traumáticas, $70,9 \%$ ocorreram em homens. A faixa etária com maior número de atendimentos foi de 21 a 30 anos. O período vespertino totalizou $23,8 \%$ das ocorrências e a região oeste do município teve a maior concentração em número de atendimentos. Os hospitais atenderam $61,2 \%$ das ocorrências. Os resultados revelaram que a equipe multiprofissional atuante no SAMU contribui significativamente na integralidade do cuidado ao ser humano.

Descritores: Ferimentos e Lesões; Serviços Médicos de Emergência; Epidemiologia.

\section{ABSTRACT}

This study aimed to characterize the care provided by the Mobile Emergency Care Service (SAMU) of Cuiabá, the capital city of Mato Grosso state, Brazil, during the first six months of operation. This is a descriptive study based on data of the SAMU'S information system service, conducted from February to June 2009. A total of 1893 calls were performed, 1293 of them for traumatic events and 578 for clinical causes. Of the latter, $51.2 \%$ occurred in females. Males accounted for $70.9 \%$ of events with traumatic causes. Most patients had 21 - to 30 -years-old . $23.8 \%$ of the attendances ocurred during the afternoon, and tThe highest frequency of attendaces occurred in the west region of the city Hospitals attended $61.2 \%$ of the SAMU's cases. The results showed that SAMU's multidisciplinary team contributes significantly to the provision of comprehensive care to patients.

Descriptors: Wounds and Injuries; Emergency Medical Services; Epidemiology.

\section{RESUMEN}

Este estudio objetivo caracterizar el atendimiento prestado por el Servicio de Urgencia de Atención Móvil de Urgencia (SAMU) de Cuiabá, capital del la provincia de Mato Grosso, Brasil, por los seis primeros meses de funcionamiento. Estudio descriptivo basado en analice documental del sistema de información del SAMU de Cuiabá, realizado en el período de febrero a junio de 2009. Fueron descriptos 1893 atendimientos, siendo 1293 debido a causas traumáticas y 578 a causas clínicas. En los atendimientos clínicos, 51,2\% fueron mujeres y en las causas traumáticas $70,9 \%$ ocurrieron en hombres. La edad con mayor numero de atendimientos fue de 21 a 30 años. El período vespertino totalizó $23,8 \%$ de las ocurrencias y la región oeste de la ciudad tuvo la mayor concentración en números de atendimientos. Los hospitales atendieran $61,2 \%$ de las ocurrencias. Los resultados muestran que la equipe multiprofesional actuante en SAMU contribuye significativamente en la integralidad del cuidado al ser humano.

Descriptores: Heridas y Traumatismos; Servicios Médicos de Urgencia; Epidemiología.

\footnotetext{
I Enfermeiro, Doutor em Ciências da Saúde. Professor Adjunto, Universidade Federal de Mato Grosso (UFMT). Cuiabá, MT, Brasil. Email: sihd@usp.br.

II Enfermeiro. Cuiabá, MT, Brasil. E-mail: bruno-oescolhido@hotmail.com.

III Matemática, Mestre em Estatística. Professor Assistente, UFMT. Cuiabá, MT, Brasil. E-mail: liamorita@ufmt.br.
} 


\section{INTRODUÇÃO}

Há evidência histórica e epidemiológica de que o nível de resposta ao atendimento de urgências e emergências tem impacto considerável na sobrevida de pessoas que apresentam complicações no estado de saúde por algum tipo de agravo, seja por causas externas (acidentes de trânsito, violência etc.) ou clínicas (principalmente relacionadas a doenças do aparelho

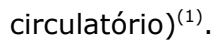

Segundo o Departamento de Informática do Sistema Único de Saúde (SUS), em 2006 as causas externas ocuparam no Brasil o terceiro lugar entre os motivos de mortalidade, com 128.388 mortes (12,4\%), ficando atrás apenas das neoplasias, com 155.796 casos fatais $(15,1 \%)$, e das doenças do aparelho circulatório, com $302.817(29,3 \%)$. Dentre as causas externas, as agressões foram responsáveis por 49.145 dos óbitos $(38,2 \%)$, seguidas de acidentes de transporte, com $37.249(29 \%)^{(2)}$.

Diante do crescente índice de mortalidade por causas externas, o Ministério da Saúde implantou em 2002 o Serviço de Atendimento Móvel de Urgência (SAMU), com a finalidade de reduzir o número de óbitos, o tempo de internação hospitalar e as sequelas decorrentes da falta de socorro precoce, garantindo assim a ampliação desse tipo de atendimento pelo SUS, considerando-se que outros grupos também prestam socorro à saúde ${ }^{(3)}$.

O SAMU dispõe de dois tipos de ambulâncias: as chamadas unidades de suporte básico, para atendimento a vítimas sem risco iminente de morte, e as unidades de suporte avançado, para casos de alto risco em emergências pré-hospitalares e transporte interhospitalar, em que são necessários cuidados médicos intensivos. No suporte básico, que não requer procedimentos de grande complexidade, a assistência pode ser realizada por profissionais de nível médio, desde que sejam qualificados, ao passo no suporte avançado as ambulâncias são estruturadas como unidades de terapia intensiva, contando com materiais e equipamentos necessários a atendimentos de maior complexidade e sendo dotadas de uma equipe composta de pelo menos três membros: médico, enfermeiro e condutor/socorrista ${ }^{(4)}$.

A implantação do SAMU em Mato Grosso $^{(5)}$ vem ao encontro das demandas por esse tipo de serviço. Em 2005, por exemplo, Cuiabá, a capital, apresentou, dentre as capitais estaduais brasileiras, a sexta maior taxa de mortalidade em homicídios, a quinta em acidentes de transporte e a sexta decorrente de causas externas ${ }^{(6)}$. Essas causas de mortalidade são frequentes nessa região federativa, com destaque para os acidentes de trânsito e as diversas formas de violência ${ }^{(7)}$.

O SAMU de Cuiabá também oferece cobertura ao município vizinho de Várzea Grande (acessível por ponte sobre o rio Cuiabá), com cerca de 300 mil habitantes, no qual permanecem uma das seis ambulâncias de suporte básico e uma das duas de suporte avançado. A central de regulação médica está sediada em Cuiabá, cuja população é de pouco mais de 500 mil.

O SAMU, no âmbito do SUS, contribui com a integralidade da assistência oferecida à população. Para atuação da equipe multiprofissional e interdisciplinar, requer-se qualificação periódica que leve em conta as especificidades de cada região brasileira. Desse modo, conhecer as principais causas dos eventos atendidos pela equipe do SAMU permite qualificar os tipos de ocorrências, embasando a implementação de políticas de saúde e de outros setores e fundamentando estratégias para promoção da saúde e prevenção de agravos.

Tendo em vista esse conjunto de aspectos, este estudo teve por objetivo caracterizar os atendimentos prestados pelo SAMU no município de Cuiabá nos seis primeiros meses de sua implantação, visando colaborar com o planejamento e a organização desse serviço oferecido à comunidade, considerando que há variáveis que determinam o tipo de assistência prestada, assim como a qualificação desejada por parte dos profissionais que prestam essa assistência.

\section{METODOLOGIA}

Trata-se de um estudo descritivo, retrospectivo, de abordagem quantitativa que utilizou dados fornecidos pelo Serviço de Atendimento Móvel de Urgência do município de Cuiabá referentes ao período de agosto de 2007 a fevereiro de 2008, correspondente a seus seis primeiros meses de operação.

Foram selecionadas somente as ocorrências atendidas pelo suporte avançado, que totalizaram 1.893 prontuários, as quais foram categorizadas em ocorrências com causas traumáticas (acidentes de trânsito em que a vítima de deslocava em moto, automóvel, bicicleta ou a pé; agressões por arma de fogo, arma branca e agressão física) e com causas clínicas (psiquiátricas; obstétricas; relacionadas aos sistemas circulatório, respiratório, digestivo e metabólico).

Os dados foram coletados de fevereiro a junho de 
2009 na sede administrativa do SAMU, utilizando-se dos prontuários de atendimento, manipulados pelos pesquisadores.

As variáveis eleitas para o estudo foram as que constavam no prontuário, categorizadas em dados de identificação da vítima (sexo e idade), tipo de ocorrência (clínica ou traumática), região do município em que o indivíduo atendido se encontrava, período do dia em que ocorreu o atendimento e local de destino do encaminhamento.

Os dados foram inicialmente dispostos em planilha elaborada para esse fim, sem necessidade de pré-teste, considerando-se que o prontuário do SAMU é padronizado.

$\mathrm{Na}$ análise, utilizou-se o programa estatístico SPSS, versão 15.0, para cálculo de frequências e porcentagens. Os resultados foram expostos na forma de estatística descritiva e organizados em tabelas.

O projeto de pesquisa foi aprovado pelo Comitê de Ética em Pesquisa Envolvendo Seres Humanos da Universidade Federal de Mato Grosso (protocolo 694/2009). Foi assinado termo de compromisso para utilização de informações de banco de dados, em atendimento à Resolução 196/96 do Conselho Nacional de Saúde ${ }^{(8)}$.

\section{RESULTADOS E DISCUSSÃO}

Nos seis primeiros meses de funcionamento do SAMU de Cuiabá (agosto de 2007 a fevereiro de 2008), as unidades de suporte avançado atenderam 1.893 pessoas no conjunto dos dois municípios de cobertura (Cuiabá e Várzea Grande).

A Tabela 1 mostra a distribuição das ocorrências atendidas, por sexo e causa.

Tabela 1: Distribuição dos atendimentos pelo Serviço de Atendimento Móvel de Urgência (SAMU) de Cuiabá, MT, por sexo e causa. Cuiabá e Várzea Grande, MT, agosto de 2007 a fevereiro de 2008.

\begin{tabular}{ccccccccc}
\hline \multirow{2}{*}{ Sexo } & \multicolumn{2}{c}{ Causa clínica } & \multicolumn{2}{c}{ Causa traumática } & \multicolumn{2}{c}{ Causa ignorada } & \multicolumn{2}{c}{ Total } \\
\cline { 2 - 9 } & $\mathbf{n}$ & $\mathbf{\%}$ & $\mathbf{n}$ & $\mathbf{\%}$ & $\mathbf{n}$ & $\mathbf{\%}$ & $\mathbf{n}$ & $\mathbf{\%}$ \\
\hline Feminino & 296 & 51,2 & 308 & 23,8 & 8 & 36,4 & 612 & 32,3 \\
Masculino & 250 & 43,3 & 916 & 70,9 & 13 & 59,1 & 1179 & 62,3 \\
Ignorado & 32 & 5,5 & 69 & 5,3 & 1 & 4,5 & 102 & 5,4 \\
\hline Total & $\mathbf{5 7 8}$ & $\mathbf{1 0 0 , 0}$ & $\mathbf{1 . 2 9 3}$ & $\mathbf{1 0 0 , 0}$ & $\mathbf{2 2}$ & $\mathbf{1 0 0 , 0}$ & $\mathbf{1 . 8 9 3}$ & $\mathbf{1 0 0 , 0}$ \\
\hline
\end{tabular}

Constatou-se que $68,3 \%$ dos atendimentos deveram-se a causas traumáticas e 30,5\% a causas clínicas. Em 5,4\% dos prontuários não havia registro dessa variável. Um estudo similar ${ }^{(1)}$, que analisou as ocorrências atendidas pelo SAMU de Olinda, PE, revelou proporções distintas, com $57 \%$ de atendimentos motivados por causas clínicas dentre um total de 1.956 ocorrências. Tomando por referência esse estudo pernambucano, evidencia-se que a população cuiabana tem comportamento mais violento, o que enseja a implementação de medidas que estimulem a cultura da $\operatorname{paz}^{(9)}$.

Embora a maioria dos atendimentos tenha sido prestada a homens $(62,3 \%)$, a estratificação por tipo de causa revela que as mulheres receberam a maioria dos atendimentos por causas clínicas $(51,2 \%)$ e que as causas traumáticas acometeram cerca de três vezes mais homens do que mulheres, evidenciando diferenças nas demandas por atendimento entre homens e mulheres. Tais achados são similares aos de um estudo realizado no Sul do Brasil ${ }^{(10)}$ que apontou altos índices de morbimortalidade masculina por acidentes e violências.

A Tabela 2 mostra a distribuição dos atendimentos por região de ocorrência, apontando um equilíbrio entre os atendimentos em Cuiabá (41\%) e Várzea Grande (40\%), justificando a necessidade desse tipo de atendimento pré-hospitalar em ambos os municípios. 
Tabela 2: Distribuição dos atendimentos pelo Serviço de Atendimento Móvel de Urgência (SAMU) de Cuiabá, MT, por região de ocorrência. Cuiabá e Várzea Grande, MT, agosto de 2007 a fevereiro de 2008.

\begin{tabular}{cccc}
\hline Região & $\boldsymbol{n}$ & \% \\
\hline Várzea Grande & 758 & 40 \\
Oeste* & 271 & 14,4 \\
Norte* & 244 & 12,9 \\
Leste* & 167 & 8,8 & 4,9 \\
Sul* & 92 & 5,4 \\
Rodovia & 103 & 13,6 \\
\hline Sem informação & 258 & $\mathbf{1 0 0}$ \\
\hline
\end{tabular}

* Regiões que compõem a área urbana de Cuiabá.

Cabe destacar que no período estudado a cidade de Várzea Grande contava com apenas uma ambulância de suporte básico, o que pode haver elevado o número dos casos menos complexos que acabaram sendo atendidos pela ambulância de suporte avançado.

Os prontuários em que não havia informações sobre o local da ocorrência representaram 13,6\% dos atendimentos, impossibilitando que se aponte com exatidão a área em que foi maior a frequência de atendimentos prestados.

Em Cuiabá, a região Oeste foi a que abarcou a maior proporção de atendimentos por causas clínicas (14,4\% do município, sendo $13,8 \%$ devidos a traumas). Essa é a região do município na qual se localiza a maioria dos acessos a Várzea Grande, onde se situa o aeroporto e por onde passam rodovias que levam ao interior do estado - vias de grande circulação que requerem medidas para a redução de acidentes, como a instalação de semáforos.

O maior número de atendimentos concentrou-se na faixa etária de 21 a 30 anos, perfazendo 34,2\% das causas traumáticas. Esse resultado é similar aos de outros estudos $^{(11-12)}$ que apontaram serem os jovens a parcela da população mais sujeita a morte por fatores externos, tendo como causa homicídio, acidente de trânsito ou suicídio.

Os atendimentos no período vespertino foram mais frequentes $(23,8 \%)$; o período noturno acumulou a menor proporção $(8,7 \%)$. Os eventos decorrentes de causas clínicas concentraram-se no período vespertino $(27,7 \%)$, enquanto os atendimentos por causas traumáticas ocorreram majoritariamente no período noturno (22,3\%). Constatou-se aumento do número de traumas no final da tarde e início da noite, período de grande circulação de veículos, devido ao retorno às residências após o turno de trabalho. O período noturno foi o de maior incidência de vítimas, uma vez que os acidentes nele ocorridos tendem a apresentar maior gravidade em decorrência de fatores como menor visibilidade, velocidade excessiva, desrespeito a semáforos e à sinalização e uso de álcool e drogas, entre outros $^{(13)}$. O horário das ocorrências não foi registrado em $13,6 \%$ das fichas de atendimento, prejudicando uma análise mais acurada dessa variável.

A Tabela 3 mostra a distribuição dos encaminhamentos dos indivíduos atendidos. Os hospitais predominaram $(61,2 \%)$, o que aponta a necessidade de se dispor de atendimento pré-hospitalar e de medidas que evitem a morte. Outro destino que chamou atenção foram os serviços psiquiátricos, que receberam $2,1 \%$ dos indivíduos atendidos. Em 1,2\% das ocorrências a vítima já falecera quando da chegada do SAMU ao local. 
Tabela 3: Distribuição do encaminhamento de indivíduos atendidos pelo Serviço de Atendimento Móvel de Urgência (SAMU) de Cuiabá, MT. Cuiabá e Várzea Grande, MT, agosto de 2007 a fevereiro de 2008.

\begin{tabular}{|c|c|c|}
\hline Destino & $n$ & $\%$ \\
\hline Ignorado & 657 & 34,7 \\
\hline Hospitais públicos em Cuiabá & 569 & 30 \\
\hline Hospitais públicos em Várzea Grande & 499 & 26,3 \\
\hline Hospitais privados em Cuiabá & 93 & 4,9 \\
\hline Recusa ao transporte & 30 & 1,6 \\
\hline Óbito & 23 & 1,3 \\
\hline Liberado no local & 16 & 0,9 \\
\hline Policlínicas & 6 & 0,3 \\
\hline Total & 1.893 & 100 \\
\hline
\end{tabular}

Constatou-se que $1,6 \%$ do total de atendidos se recusaram a ser encaminhados após receberem atendimento pela equipe do SAMU. Tal situação limita o atendimento pelo serviço aos que necessitam apenas de atendimento pré-hospitalar, além de levar ao desgaste físico e emocional da equipe.

Outro achado relevante foi o alto percentual de fichas de atendimento sem registro de encaminhamento $(34,7 \%)$. Tais registros são utilizados em perícias por empresas de seguro, além de proporcionarem informações legais de interesse para a vítima, sua família e a polícia, quando há necessidade de investigações. Essa lacuna é agravada pelo fato de o SAMU não dispor de um sistema informatizado que permita 0 armazenamento de dados e informações sobre os atendimentos prestados ${ }^{(14)}$.

A Tabela 4 mostra a distribuição dos atendimentos segundo o tipo e motivo das ocorrências.

Tabela 4: Distribuição dos atendimentos pelo Serviço de Atendimento Móvel de Urgência (SAMU) de Cuiabá, MT, por tipo e motivo da ocorrência. Cuiabá e Várzea Grande, MT, agosto de 2007 a fevereiro de 2008.

\begin{tabular}{cccc}
\hline Tipo de ocorrência & Motivo da ocorrência & $\boldsymbol{n}$ & \% \\
\hline \multirow{2}{*}{ Causas traumáticas } & Acidentes de trânsito & 633 & 68,4 \\
& Agressões (arma de fogo, branca e agressão física) & 293 & 31,6 \\
\cline { 2 - 4 } Causas clínicas & Total & $\mathbf{9 2 6}$ & $\mathbf{1 0 0}$ \\
\cline { 2 - 4 } & Relacionado aos sistemas corporais & 341 & 62,5 \\
& Psiquiátrico & 23,9 \\
\cline { 2 - 4 } Informação prejudicada & Obstétrico & 130 \\
\cline { 2 - 4 } & Total & $\mathbf{7 4}$ & $\mathbf{1 0 0}$ \\
\cline { 2 - 4 } & Ignorado (sem registro) & 407 & 96,4 \\
\cline { 2 - 4 } Total & Ilegível & 15 & 3,6 \\
\hline
\end{tabular}

Os acidentes envolvendo motocicletas foram responsáveis por $29,8 \%$ dos que ocasionaram trauma, constituindo a maioria desse tipo de ocorrência. Estudo similar realizado em Catanduva, SP(15), apontou que de 1.486 fichas de vítimas de trauma, 42,2\% mencionavam acidente motociclístico. No presente estudo os acidentes com esse tipo de veículo superaram os ciclísticos e automobilísticos, confirmando a maior propensão das motocicletas a acidentes de trânsito.

Esses achados reforçam a necessidade de atenção por parte do sistema governamental para a melhoria da segurança no trânsito, o que inclui a sinalização de vias, instalação de semáforos e policiamento.

As agressões totalizaram $31,6 \%$ das ocorrências devidas a trauma, $12 \%$ dos quais envolveram arma de fogo. Tais óbitos, originados de situações preveníveis, geram alto custo para as esferas governamentais e desestruturação familiar e social, além de afetarem o mercado de trabalho ${ }^{(4)}$.

As ocorrências de origem psiquiátrica tiveram a maior proporção entre os atendimentos motivados por causas clínicas (23,9\% das ocorrências), seguidas das urgências gineco-obstétricas $(13,6 \%)$ e doenças do aparelho circulatório $(13,3 \%)$. 
Em decorrência das políticas de desinstitucionalização do doente mental, houve um aumento da demanda aos serviços de emergência psiquiátrica ${ }^{(15)}$, o que é corroborado por nossos resultados. As demais causas clínicas somaram 34,9\% das ocorrências, sendo que as convulsões representaram 9,9\%, seguidas das intoxicações exógenas, com 8,3\%, e das síncopes, com 3,8\%.

Chamamos atenção para o percentual de informações prejudicadas nas fichas de atendimento $(22,3 \%)$, o que revela uma fragilidade da equipe no registro das informações. Embora seja esperado um percentual de informações ignoradas, já que a ação da equipe deve ser rápida e priorizar o atendimento ao paciente, as lacunas e imprecisões no preenchimento prejudicam a integridade dos registros. É recomendado que a equipe preencha todos os dados dos prontuários para que estes se prestem a levantamentos subsequentes, contribuindo para o delineamento e monitoramento do perfil das ocorrências, entre outros usos.

\section{REFERÊNCIAS}

1. Cabral APS, Souza WV. Serviço de Atendimento Móvel de Urgência (SAMU): análise da demanda e sua distribuição espacial em uma cidade do Nordeste brasileiro. Rev. bras. epidemiol. [Internet]. 2008 [cited 2011 set 30];11(4):530-40. Available from: http://www.scielo.br/pdf/rbepid/v11n4/01.pdf.

2. DATASUS [Internet]. Brasília: Ministério da Saúde (BR) [cited 2011 set 30]. Departamento de Informática do SUS - DATASUS. Available from: http://www2.datasus.gov.br/DATASUS/index.php?area $=02$. 3. Ministério da Saúde. Portaria no 2048 GM/MS de 5 de novembro de 2002. Dispõe sobre a regulamentação do atendimento das urgências e emergências. Brasília (Brasil): Ministério da Saúde; 2002.

4. Sanches S, Duarte SJH, Pontes ERJC. Caracterização das vítimas de ferimentos por arma de fogo, atendidas pelo Serviço de Atendimento Móvel de Urgência em Campo Grande-MS. Saude soc. [Internet]. 2009 [cited 2011 set 30];18(1):95-102. Available from: http://www.scielo.br/pdf/sausoc/v18n1/10.pdf. 5. Ministério da Saúde. Portaria no 2300 GM/MS de 18 de setembro de 2007. Dispõe sobre a habilitação do Serviço de Atendimento Móvel de Urgência - SAMU 192 do Estado de Mato Grosso, localizado no município de Cuiabá (MT). Brasília (Brasil): Ministério da Saúde; 2007.

6. Oliveira LR, Jorge MHPM. Análise epidemiológica das causas externas em unidades de urgência e emergência em Cuiabá/Mato Grosso. Rev. bras. epidemiol. [Internet]. 2008 [cited 2011 set 30];11(3):420-30. Available from: http://www.scielo.br/pdf/rbepid/v11n3/08.pdf.

7. Marchese VS, Scatena JHG, Ignotti E. Caracterização das vítimas de acidentes e violências atendidas em serviço de emergência: Município de Alta Floresta, MT (Brasil). Rev. bras. epidemiol. [Internet]. 2008 [cited 2011 set 30];11(4):648-59. Available from: http://www.scielo.br/pdf/rbepid/v11n4/11.pdf.

8. Ministério da Saúde; Conselho Nacional de Saúde. Resolução No 196/96 - Normas regulamentadoras de pesquisa envolvendo seres humanos. Brasília (Brasil): Ministério da Saúde; 1996.

9. Nelman J, Ciliberti ME; Aoki M, Figueira Junior N. Tecendo

\section{CONCLUSÃO}

Uma limitação deste estudo está na população focalizada, que não abrange a totalidade dos indivíduos atendidos pelo SAMU de Cuiabá, uma vez que não foram incluídos os atendimentos realizados pelas unidades de suporte básico e tampouco os prestados por outros serviços nessa localidade, o que pode constituir objeto de outras pesquisas.

A caracterização do perfil de atendimento do SAMU de Cuiabá permitiu equacionar os principais problemas de saúde atendidos, contribuindo para o planejamento de ações intersetoriais de promoção à saúde, incluindo dimensionamento de pessoal e alocação e otimização de recursos, além de subsidiar decisões estratégicas úteis à esfera gestora municipal.

Este estudo permite sugerir que os treinamentos das equipes do SAMU de Cuiabá priorizem aspectos referentes à cinemática do trauma, incluindo a remoção de vítimas em quadros de difícil acesso e procedimentos orientados pelo $A B C D E$ do trauma, além de procedimentos voltados a indivíduos com transtornos mentais. Esperamos que os resultados alcançados impulsionem a realização de novos estudos.

redes de paz. . Saude soc. [Internet]. 2009[cited 2011 set 30];18(1):66-72. http://www.scielo.br/pdf/sausoc/v18s1/12.pdf 10. Sant'anna A, Aerts D, Lopes MJM. Homicídios entre adolescentes no sul do Brasil: situações de vulnerabilidade segundo seus familiares. Cad Saude Publica [Internet]. 2005 [cited 2011 set 30];21(1):120-9. Available from: http://www.scielo.br/pdf/csp/v21n1/14.pdf.

11. Máximo W. Mortalidade de jovens leva Brasil ao terceiro lugar em homicídios entre 84 países [Internet]. Florianópolis: Eco \& Ação; 2006 nov 18 [cited 2011 set 30]. Available from: http://www.ecoeacao.com.br/index2.php?option=com_content\&d o_pdf $=1 \& i d=307$.

12. Cocco M, Lopes MJM. Morbidades por causas externas em adolescentes de uma região do município de Porto Alegre. Rev. Eletr. Enf. [Internet] 2010 [cited 2010 set 30];12(1):89-97. Available

http://www.fen.ufg.br/revista/v12/n1/pdf/v12n1a11.pdf.

13. Batista SEA, Baccani JG, Silva RAP, Gualda KPF, Viana Junior RJA. Análise comparativa entre os mecanismos de trauma, as lesões e o perfil de gravidade das vítimas, em Catanduva - SP. Rev Col Bras Cir [Internet]. 2006 [cited 2011 set 30];33(1):610. Available from: http://www.scielo.br/pdf/rcbc/v33n1/v33n1a02.pdf.

14. Marques CQ, Lima MADS, Ciconet RM. Agravos clínicos atendidos pelo Serviço de Atendimento Móvel de Urgência (SAMU) de Porto Alegre - RS. Acta paul. enferm. [Internet]. 2011 [cited 2011 set 30];24(2):185-91. Available from: http://www.scielo.br/pdf/ape/v24n2/05.pdf.

15. França Neto O Uma ética para os novos desdobramentos da reforma psiquiátrica. Ágora (Rio J.) [cited 2011 set 30]. 2009 [cited 2011 set 30];12(1):119-29. Available from: http://www.scielo.br/pdf/agora/v12n1/08.pdf.

Artigo recebido em 17.08.2010.

Aprovado para publicação em 08.08.2011.

Artigo publicado em 30.09.2011. 